\title{
Silvina Chemen, Francisco Canzani, Un dialogo para la vida, Madrid: Editorial Ciudad Nueva, 2017, pp. 236 [Buenos Aires: Editorial Ciudad Nueva, 2013; $A$ dialogue of life, New York: JTS-New City Press, 2015]
}

DOI: http://dx.doi.org/10.12775/PCh.2019.037

Ho appena letto un libro edito da Ciudad Nueva argentina nel 2013, ristampato dall' editrice spagnola nel 2017. Un libro che solo dal titolo $U n$ dialogo per la vita, invita alla lettura. Il sottotitolo Verso l'incontro tra giudei e cristiani centra più specificamente l'argomento mentre i nomi degli autori sono corredati da questa frase: A due voci e all'unisono.

E chi sono questi autori? Ecco qui un altro richiamo: una rabbina ebrea e un avvocato cristiano cattolico, "le voci all' unisono", autori di un testo prezioso per la formazione al dialogo, e non solo quello ebraico-cristiano, perché in queste pagine essi testimoniano l'arte del dialogo vissuto ad ampio raggio nella propria vita.

Nell'oggi del mondo, la parola dialogo ha un valore aggiunto: chi non ha mai avuto l'esigenza, il bisogno e il desiderio di un dialogo vero? Ma in questa società, vedendo come scorrono gli avvenimenti, i conflitti, i malintesi, il dialogo sembra essere, a volte, un'autentica utopia. E perciò parlare di dialogo è già un appello. Inoltre, un "dialogo per la vita", come ci annuncia il libro, invita in modo accattivante il lettore ad aprire subito le sue pagine.

Gli autori guardano in faccia le sfide di un dialogo interreligioso vero e lo fanno in modo vitale, partendo dalla vita, o meglio, dalla propria vita ed esperienza vissuta insieme, in un cammino di dialogo sincero appartenendo a religioni, mondi e contesti molto diversi.

Con un'attenta pedagogia, ci aprono vie per un dialogo tra giudei e cristiani - e non solo tra loro - un dialogo rispettoso delle proprie identità e differenze senza mancare affatto di profondità e verità.

Il libro è unico in questo senso, perché nasce dal dono e dalla conoscenza reciproca di due persone straordinariamente sagge e sensibili - come dice nel prologo il rabbino Jack Bemporad - che aprono nuove vie di conoscenza tra due comunità che possono lavorare insieme imparando gli uni dagli altri, alla ricerca di un fondamento comune per affrontare insieme gli attuali problemi etici e politici.

Una significativa frase del libro dà un' idea della vitalità del testo in questione: "il dialogo si sviluppa non solo per via dei documenti o nelle di- 
chiarazioni delle più autorevoli autorità. Sono passi essenziali, ma il dialogo non viene recitato o imposto, ma scaturisce, e nasce dalla volontà degli esseri umani che, essendo stati separati, decidono di provare a costruire ponti di scambio" (p. 39).

$\mathrm{Ci}$ sono, infatti, nel testo espressioni che aprono orizzonti di un pensare tanto necessario oggi: "il dialogo è uno stile di vita e un modo di guardare l'altro e il mondo"; "la storia dell'altro"; "la fiducia"; "lo sguardo positivo"; "l'onestà intellettuale e l'onestà spirituale". E via via concetti e riflessioni che attirano e suscitano tante domande nel lettore.

Personalmente e come pedagogista, sottolineo l'importanza di un approccio al dialogo da questo punto di vista tanto da poter parlare di pedagogia del dialogo. Dicono gli autori: "Non siamo pedagoghi, ma persone che hanno il titolo di «apprendisti e moltiplicatori di dialogo» perché abbiamo preso la decisione vitale di osare all'avventura dell'incontro con l'altro" (p. 18). E qui forse è da sottolineare l'intenzione esplicita del libro, che gli autori considerano un testo formativo, specifico per la formazione al dialogo, definendolo uno

strumento per il dialogo. Queste pagine sono scritte con l'intenzione di offrire strumenti accessibili a tutti in modo che possano contribuire, quando applicati, all'incontro tra persone di diverse religioni e culture. Strumenti che possono essere utili in particolare a coloro che svolgono compiti educativi nelle comunità (formatori, catechisti, maestri ed insegnanti) e che servono all'interno della famiglia, lo spazio privilegiato per la formazione di uomini e donne capaci di dialogo (p. 20).

Gli autori hanno ben definito anche i destinatari del loro lavoro: "Questo libro è stato scritto pensando a tutti gli uomini e le donne che nei loro molteplici campi sviluppano compiti educativi, sia formali che informali; all'interno di comunità o parrocchie, nelle scuole, nei gruppi giovanili, nei movimenti religiosi, ricreativi, culturali o universitari" (p. 47). Comunque possiamo dire che in questa definizione possono rientrare tutti gli esseri umani perché, ad essere sinceri, anche chiunque si trovi a vivere in contesti di rapporti umani, pur non avendo specifici compiti educativi non può eludere di fatto la responsabilità di un compito educativo informale: in un certo senso "tutti siamo allievi e maestri".

Nel volume si parla anche dell'utilità del dialogo. Gli autori chiariscono - in modo definitivo e sublime con una frase significativa - quale sia la sola finalità di approdarvi: "Il dialogo non ha uno scopo utilitario, ma perse- 
gue un obiettivo trascendente. Io non lo faccio perché otterrò questo o quel beneficio, ma il fatto stesso di dedicarmi ad esso conferisce dignità alla mia esistenza" (p. 165).

Molto interessante la definizione dell'arte della domanda e dell'ascolto e di come imparare a domandare e ad ascoltare, una pratica tanto infrequente oggi.

E nella parte finale del libro è quanto meno interessante, se non imprescindibile, l'approfondimento di quello che gli autori definiscono gli ostacoli al dialogo, la cui lettura apre gli occhi su una nuovissima dimensione del dialogo.

Non è facile chiudere questa recensione perché ci sarebbero ancora parecchie sfumature da sottolineare, ma lasciamo che siano i lettori stessi a scoprirle, nella speranza di averli entusiasmati a iniziare insieme agli autori l'avventura di un "dialogo per la vita".

Araceli Del Pozo Armentia* ORCID: 0000-0002-0088-5609

Madrid, España

\section{Silvina Chemen, Francisco Canzani, Un dialogo para la vida \\ [Dialog życia], Madrid: Editorial Ciudad Nueva, 2017, s. 236 [Buenos Aires: Editorial Ciudad Nueva, 2013; A dialogue of life, New York: JTS-New City Press, 2015]}

DOI: http://dx.doi.org/10.12775/PCh.2019.038

Książka wydana w języku hiszpańskim przez argentyńskie wydawnictwo Ciudad Nueva w 2013, a potem w 2017 r. przez to samo wydawnictwo w Hiszpanii już samym tytułem Dialog życia (Un dialogo para la vida) zachęca do lektury. Podtytuł Ku spotkaniu żydów i chrześcijan wskazuje na podjęte w niej zagadnienia, przy nazwiskach autorów dodano zaś takie okre-

* Araceli Del Pozo Armentia, Professore di psicopedagogia all' Universidad Complutense de Madrid - España e di pedagogia all'Istituto Universitario Sophia - Italia. Indirizzo: Universidad Complutense de Madrid, C/ Rector Royo Villanova 1, Ciudad Universitaria 28040 Madrid. Mail: apa@ucm.es. 